\title{
Norois
}

Environnement, aménagement, société

$240 \mid 2016$

Aménagement foncier, risque côtier, évolution des fleuves

\section{Submersion. Comment gérer la montée du niveau des mers, Laurent Labeyrie}

\section{Yannick Lageat}

\section{(2) OpenEdition}

\section{Journals}

Édition électronique

URL : http://journals.openedition.org/norois/5967

DOI : 10.4000 /norois.5967

ISBN : 978-2-7535-5362-0

ISSN : $1760-8546$

\section{Éditeur}

Presses universitaires de Rennes

\section{Édition imprimée}

Date de publication : 30 novembre 2016

Pagination : 102-104

ISBN : 978-2-7535-5356-9

ISSN : 0029-182X

\section{Référence électronique}

Yannick Lageat, "Submersion. Comment gérer la montée du niveau des mers, Laurent Labeyrie ", Norois [En ligne], 240 | 2016, mis en ligne le 30 novembre 2016, consulté le 23 septembre 2020. URL : http:// journals.openedition.org/norois/5967 ; DOI : https://doi.org/10.4000/norois.5967 
les contextes politiques, entre fermeture et ouverture à tous les flux, matériels ou non.

Pour sécuriser un territoire devenu brutalement vulnérable, les politiques américains ont défini un concept d'un nouveau genre, celui de « frontière intelligente ». Le $49^{\mathrm{e}}$ parallèle est passé du statut d'une des plus longues frontières démilitarisées du monde à celui d'une « barrière hautement sécurisée ». L'auteur analyse donc le contenu de cette frontière intelligente, tout en évaluant son degré de réussite sur les deux dimensions d'une frontière, la sécurité et le développement des échanges. Il dégage le jeu et l'imbrication des forces et des stratégies qui organisent le fonctionnement de cette frontière intelligente. S'attachant à démystifier la frontière, entre autres par une étude des coûts de "l'intelligence », P.-A. Beylier nous renseigne au final sur l'ambigüité de la relation américano-canadienne, à la fois «familiale» mais aussi source de soupçons plus ou moins formulés sur l'inégalité du partenariat bilatéral.

Ce livre très informé, à l'écriture soutenue, abondamment appuyé par le recours aux cartes et aux photographies, stimulera encore plus les réflexions sur les nouvelles constructions géopolitiques d'un monde devenu à la fois plus partenarial et interconnecté mais aussi plus instable que jadis.

Christian Pinet Université d'Angers

Laurent LabeYrie, 2015. Submersion. Comment gérer la montée du niveau des mers, Paris, Odile Jacob, 164 p.

Cet ouvrage retrace le développement des connaissances sur les changements du niveau marin au cours du dernier demi-siècle, sur lesquelles, soucieux des « conséquences pour la population du globe », il s'appuie pour hasarder « une appréciation raisonnable » de son évolution probable dans le futur. La quatrième de couverture donne le ton : « La mer monte... Inéluctable effet du réchauffement climatique, cette élévation du niveau moyen des mers n'est que de quelques millimètres par an : rien là de bien spectaculaire, même si cela suffit, comme à La Faute-sur-Mer, pour causer de terribles catastrophes. Qu'en sera-t-il lorsqu'une montée des eaux de plus d'un mètre sera atteinte d'ici à la fin du siècle, et bien plus par la suite? »

Faut-il croire que la submersion des 27-28 février 2010 est plus imputable à l'élévation séculaire, évaluée à $21 \mathrm{~cm}$ dans le pertuis d'Antioche depuis 1850 $\left(\right.$ T. Gouriou $\left.{ }^{1}\right)$, qu'à la surcote exceptionnelle de $153 \mathrm{~cm}$ qui y a été mesurée? Décidément, Xynthia aura beaucoup servi à donner du crédit (et des crédits) au catastrophisme environnemental. Osons rappeler que la différence positive entre le niveau réellement observé et le niveau prédit de la marée

1. Évolution des composantes du niveau marin à partir d'observations de marégraphie effectuées depuis la fin du XVIII siècle en Charente-Maritime, thèse d'océanographie physique, Université de La Rochelle, 2012, 492 p.

\section{LAURENT LABEYRIE}

\section{SUBMERSION COMMENT GÉRER LA MONTÉE DU NIVEAU DES MERS}
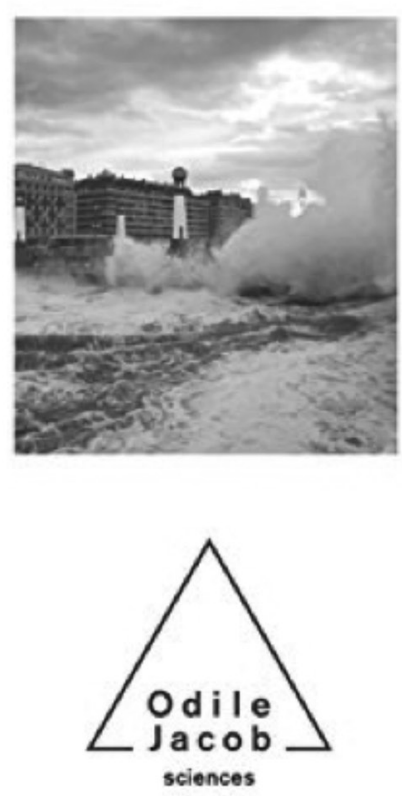
résulte d'une conjonction aléatoire : il n'est que de relire les travaux sur le littoral atlantique de Paolo A. Pirazzoli ${ }^{2}$ qui n'est cité qu'à propos des Champs Phlégréens!

Comme l'a judicieusement noté Jean-René Vanney $^{3}$, des «surcotes d'origine tempétueuse [sont] métamorphosées en prémices de la submersion inéluctable à venir. » Il est évident que les deux phénomènes, même s'ils s'entremêlent, ne relèvent pas des mêmes échelles spatio-temporelles qui n'offrent pas les mêmes possibilités de modélisation. En l'occurrence, il serait heureux que l'inquiétude se portât autant sur la survenance imprévisible d'événements météorologiques extrêmes que sur la hausse programmée du niveau de la mer, laquelle tend malencontreusement à cautionner l'imprévision dans la prise en charge du risque de submersion.

Le dernier rapport du GIEC, datant de 2014, a livré la fourchette suivante à l'échéance de la fin du $\mathrm{XXI}^{\mathrm{e}}$ siècle : + 0,28 à $+0,98 \mathrm{~m}$, la valeur la plus probable se situant autour de $44 \mathrm{~cm}$. S'additionneraient l'expansion thermique des eaux océaniques de surface pour $26 \mathrm{~cm}$, la fonte des glaciers de montagne pour $17 \mathrm{~cm}$, celle du Groenland pour $9 \mathrm{~cm}$ et celle du pergélisol - ou permafrost - pour $1 \mathrm{~cm}$, soit un total de $53 \mathrm{~cm}$, mais l'accroissement attendu des précipitations neigeuses sur l'Antarctique le diminuerait de $9 \mathrm{~cm}$. La vitesse d'élévation du niveau marin pourrait donc être trois fois supérieure à celle estimée pour le $\mathrm{xx}^{\mathrm{e}}$ siècle, mais le comportement des glaces polaires face à l'effet de serre additionnel est l'objet de controverses. Ainsi, tenant compte de l'accélération du glissement des glaces du Groenland et de l'Antarctique occidental dans la mer, les derniers travaux de l'université de Californie à Irvine situent l'augmentation prévisible du niveau marin entre 50 et $150 \mathrm{~cm}$ en 2100 , avec des disparités régionales importantes, dues à des phénomènes géologiques locaux, sinon régionaux ${ }^{4}$. Largement médiatisé, un récent article de Robert M. DeConto et David Pollard ${ }^{5}$ s'inquiète d'une possible déstabilisation

2. «Surges, atmospheric pressure and wind change and flooding probability on the Atlantic coast of France ", Oceanologica Acta, vol. 23, 2000, n 6 , p. $643-661$.

3. "Le relèvement contemporain du niveau moyen de l'Océan ", Annuaire du Droit de la Mer, t. V, 2000, p. 295-344.

4. Carson M. et al., "Coastal sea level changes, observed and projected during the $20^{\text {th }}$ and $21^{\text {st }}$ century ", Climatic Change, vol. 134, 2016, $n^{\circ} 1-2$, p. 269-281.

5. "Contribution of Antarctica to past and future sea-level rise ", Nature, vol. 531, 2016, p. 591-597. de la calotte ouest-antarctique qui disposerait d'un potentiel d'élévation d'un mètre en 2100 et même de 15 m à l'échéance de 2500, si les émissions de gaz à effet de serre se prolongeaient au rythme actuel (selon le scénario Business as Usual).

Évidemment, les chiffres produits par Laurent Labeyrie dans sa préface ne peuvent qu'effrayer le lecteur alerté par une élévation qui «pourrait d'ici un siècle dépasser 3 à 5 mètres » (p. 7), et la conclusion ne saurait le tranquilliser : « Les grandes marées et les tempêtes monteront toujours plus haut, pour s'approcher du mètre à la fin du siècle, et de 2 à 5 mètres ou plus dans les siècles suivants » (p. 145). On barbotte à nouveau en pleine confusion à propos du risque associé à des événements météomarins ponctuels : l'auteur ne devrait pas ignorer qu'une «marée du siècle », comme celle du 21 mars 2015, a pu décevoir le spectateur avide de sensations fortes, alors que l'ouragan du 15 octobre 1987 engendrait des dégâts considérables sur les côtes bretonnes par morte-eau...

Accoutumé au fil des pages à une succession d'approximations, on ne s'étonnera pas d'apprendre que « des calottes de glace descendaient jusqu'en Belgique » (p. 19), et qu'en Hollande, « les glaciers et les dépôts sédimentaires associés ont sculpté la plaine jusqu'au niveau de la mer sur presque deux tiers du territoire [sic], créant de grandes surfaces marécageuses » (p. 21). Encore faut-il d'ailleurs que l'extraction de la tourbe y ait créé des étangs pour que les habitants aient pris la décision de les endiguer!

Passant charitablement sur les développements climatologiques, on n'est pas plus surpris que la surface de la Terre soit modelée par un ensemble de processus « qui tend à l'aplanir : l'érosion et le transport par les vents, les glaces, les rivières, les racines des plantes, les vagues et les courants marins » (p. 23), et on retiendra, entre autres curiosités, que, «dans les climats tropicaux, les atolls de corail marquent les anciens cratères submergés » (p. 27). On est en droit d'espérer que la mesure du $\delta^{18} \mathrm{O}$ ne souffre pas d'autant d'imprécisions...

On aurait pu attendre de sa toute récente expérience d'édile à Arzon que l'auteur disposât d'une connaissance approfondie de son nouveau terrain d'élection. Jugeons-en! Dans le golfe du Morbihan, « de grandes vasières [...] sont les restes de prairies, transformées au cours des derniers milliers d'années par les processus naturels en "prés-salés” » (p. 26-27). 
Il est vrai que «l'enfoncement de la Bretagne-Sud d'environ $1 \mathrm{~mm} / \mathrm{an}[\ldots]$ provoque une montée correspondante du niveau marin local » (p. 101), tandis qu'« en Bretagne-Nord, le sol a tendance à se soulever progressivement, de 1 à $2 \mathrm{~mm} / \mathrm{an}$. La montée moyenne du niveau de la mer est en gros compensée par ce mouvement » (p. 110). Confronté à ces taux calabrais ou corinthiens, le géomorphologue se demande à quel interstade du Würm doivent désormais être rapportés les vestiges des « plages soulevées normanniennes » observables en Léon et en Trégor!

Quant aux propositions de gestion, sur lesquelles le sous-titre appelle l'attention, elles ne se signalent guère par leur audace, et on retiendra que " la réponse à la montée du niveau de la mer et au changement climatique ne peut être approchée de façon dogmatique » (p. 133) et qu'il faut encourager le passage aux énergies non carbonées.

Plus intéressants que ceux qui le lient à sa tante Henriette ou à la Société amicale des géologues amateurs, les souvenirs des campagnes océanographiques ou des recherches menées au Centre des faibles radioactivités de Gif-sur-Yvette peuvent être lus sans ennui. On n'ignore pas que Laurent Labeyrie est le co-auteur de travaux de référence sur les anomalies isotopiques des foraminifères planctoniques fossiles. Par respect pour sa contribution à la connaissance des paléo-environnements, on oubliera, sitôt refermé, cet ouvrage dont la lecture est fortement déconseillée. Le géomorphologue, dont l'expertise n'est sollicitée qu'à propos d'une description confuse d'un tombolo (p. 118), n'y apprendra rien qu'il ne sache déjà s'il est familier de l'histoire des littoraux et a fortiori de leur dynamique présente, à laquelle sont dévolus quelques développements bizutaux (p. 12-26).

Yannick LAGEAT Université de Bretagne Occidentale

\section{André Siegfried, 2016. Le tableau politique de la France de l'Ouest. 100 ans après, héritages et postérités, Rennes, PUR, coll. «Géographie sociale », 350 p.}

Aboutissement d'un colloque tenu à Cerisy en 2013 à l'occasion du centenaire de la publication du Tableau politique de la France de l'Ouest, cet ouvrage opère un retour bienvenu sur l'œuvre fondatrice d'André Siegfried. Il réunit dix-huit contributions mariant avec bonheur les points de vue de la géographie sociale et de la science politique. Pour des raisons très conjoncturelles, cette dernière s'est explicitement attachée à ériger la figure d'André Siegfried en père fondateur et à faire du Tableau... une œuvre pionnière. Mais la science politique a-telle pour autant contribué à faire connaître l'homme et son œuvre? Connu de tous les apprentis politologues, cité en ouverture de tous les manuels, André Siegfried est pourtant peu lu. Souvent caricaturé (« le calcaire vote à gauche, le granit à droite»), le fondateur de la sociologie électorale est comme éclipsé par sa notoriété. Il fallait le relire, risquer de remettre en discussion ses méthodes et ses hypothèses, bref oser une critique scientifique à laquelle la panthéonisation ne prédispose guère. C'est chose faite avec cet ouvrage qui suit deux pistes complé- mentaires : la première consiste à utiliser les outils des sciences sociales pour comprendre la production singulière d'une œuvre et pour comprendre surtout comment elle a pu acquérir le statut de classique, voire de texte fondateur; la seconde tente de revenir sur les principes fondateurs de la méthode empruntée par l'auteur du Tableau... pour évaluer dans quelle mesure celle-ci permet encore de comprendre les évolutions de la carte électorale. Expliquer Siegfried d'un côté, prolonger Siegfried de l'autre.

André Siegfried était paraît-il étonné de constater à la fin de sa vie que ce qu'il considérait comme une œuvre de jeunesse, œuvre au demeurant assez isolée au regard de ses autres publications, ait pu de la sorte acquérir le statut de classique. Rappelons avec Alain Garrigou que son père, importateur de coton au Havre, grande figure de la bourgeoisie protestante, fut un homme politique important (ministre du Commerce); qu’André Siegfried lui-même tenta à plusieurs reprises de devenir député, et que l'insuccès fut l'un des moteurs de sa curiosité électo- 